\title{
Mechanical Properties of Direct Waste Printing of Polylactic Acid with Universal Pellets Extruder: Comparison to Fused Filament Fabrication on Open-Source Desktop Three-Dimensional Printers
}

\author{
Arthur Alexandre, ${ }^{1}$ Fabio A. Cruz Sanchez, ${ }^{1}$ Hakim Boudaoud, ${ }^{1}$
} Mauricio Camargo, ${ }^{1}$ and Joshua M. Pearce ${ }^{1,2,3}$

\begin{abstract}
Fused filament fabrication (FFF) is the most common and widespread additive manufacturing (AM) technique, but it requires the formation of filament. Fused granular fabrication (FGF), where plastic granules are directly three-dimensional (3D) printed, has become a promising technique for the AM technology. FGF could be a key driver to promote further greening of distributed recycling thanks to the reduced melt solidification steps and elimination of the filament extruder system. However, only large-scale FGF systems have been tested for technical and economic viability of recycling plastic materials. The objective of this work is to evaluate the performance of the FFF and FGF techniques in terms of technical and economical dimensions at the desktop 3D printing scale. Recycled and virgin polylactic acid material was studied by using five different types of recycling feedstocks: commercial filament, pellets, distributed filament, distributed pellets, and shredded waste. The results showed that the mechanical properties from the FGF technique using same configurations showed no statistical differences to FFF samples. Nevertheless, the granulometry could have an influence on the reproducibility of the samples, which explains that the critical factor in this technology is to assure the material input in the feeding system. In addition, FGF costs per $\mathrm{kg}$ of material were reduced to less than $1 € / \mathrm{kg}$ compared with more than $20 € / \mathrm{kg}$ for commercial recycled filament. These results are encouraging to foster FGF printer diffusion among heavy users of 3D printers because of reducing the cost associated to the filament fabrication while ensuring the technical quality. This indicates the possibility of a new type of 3D printing recycled plastic waste that is more likely to drive a circular economy and distributed recycling.
\end{abstract}

Keywords: fused granular fabrication, recycling, polylactic acid, tensile properties, distributed recycling

\section{Introduction}

Plastics ARE AMONG the most abundant materials in our industrial environment and recycling has long been established as the optimum postconsumer treatment of plastic waste. ${ }^{1}$ However, this valuable resource ${ }^{2}$ is often wasted as only $9 \%$ of plastic waste generated has been recycled. ${ }^{3}$ If current centralized plastic waste management trends con- tinue, 12,000 Mt of plastic waste will be landfilled or dispersed in the environment polluting it by mid-century. ${ }^{3}$ Centralized recycling has a high embodied energy (particularly from transportation and sorting). ${ }^{4,5}$

Fortunately, the rise of economically competitive distributed manufacturing with three-dimensional (3D) printers ${ }^{6-10}$ offers the potential to manufacture products close to consumers and even in their own homes with reduced

\footnotetext{
${ }^{1}$ ERPI, Université de Lorraine, F-54000 Nancy, France.

Departments of ${ }^{2}$ Materials Science \& Engineering and ${ }^{3}$ Electrical \& Computer Engineering, Michigan Technological University, Houghton, Michigan, USA.

(C) Alexandre et al. 2020; Published by Mary Ann Liebert, Inc. This Open Access article is distributed under the terms of the Creative Commons Attribution Noncommercial License (http://creativecommons.org/licenses/by-nc/4.0/) which permits any noncommercial use, distribution, and reproduction in any medium, provided the original author(s) and the source are cited.
} 
environmental impacts. ${ }^{11,12}$ The development of the opensource waste plastic extruder that produces filament for $3 \mathrm{D}$ printing (recyclebot) ${ }^{13}$ offers the potential for distributed recycling ${ }^{14}$ with an improved environmental ${ }^{11,15-17}$ as well as economic performance. ${ }^{18}$ Due to the introduction of the open-source self-replicating rapid prototyper (RepRap), ${ }^{19-21}$ the dominant technology of 3D printing is fused filament fabrication (FFF) using polylactic acid (PLA). ${ }^{22}$ Various forms of filament extrusion systems have proven effective at recycling PLA. ${ }^{14,23-26}$ However, PLA degrades with each cycle through the print/grind/extrude to filament/print loop. ${ }^{14,23,27}$ This issue can be partially controlled by adding virgin PLA to recycled PLA, ${ }^{14,23}$ coatings, ${ }^{17}$ or carbon fiber reinforcement. ${ }^{28}$

In addition, material extrusion $3 \mathrm{D}$ printers that can print directly from pellets of plastic have been developed ${ }^{29-33}$ and commercialized (e.g., David, Erecto-Struder, GigabotX, Cheetah Pro, Part Daddy). This type of 3D printer offers the potential to reduce the melt cycles in the fabrication of 3Dprinted parts made from recycled plastic materials. In addition to pellets, a large-format prototype GigabotX 3D printer has printed from flakes of plastic, regrind, or shreds of recycled plastic (or polymer "particles") (thus called fused particle fabrication) or fused granular fabrication (FGF). ${ }^{24}$ This method of 3D printing not only supports direct recycling of waste but can also be economically competitive at the industrial machine distributed manufacturing level (e.g., at a fablab). ${ }^{34}$ Some of the advantages of eliminating the use of filament are the reduction in the cost of materials, the increase in the number of types of polymers that can be recycled, the reduced embodied energy from eliminating a melt cycle, and the reduction in production time, eliminating the time required to manufacture filament. $^{24,34,35}$

These direct waste commercial 3D print systems are all large and meant for localized (not home) distributed manufacturing.
However, there have been several attempts at making smallscale pellet extruders ${ }^{36-38}$ and one company (Mahor XYZ) has begun to market kits ${ }^{39}$ to convert conventional fused filament $3 \mathrm{D}$ printers to fused pellet machines. In this study, these kits are used to determine the technical viability of using direct material extrusion 3D printing on the small scale to recycle PLA (the most common desktop 3D-printed material). Specifically, the objective of this work was to evaluate and compare technical and economic aspects of two types of open-source 3D desktop printers: (1) an FFF and (2) an FGF using virgin and recycled PLA. The results are discussed to determine the potential for distributed recycling by using desktop 3D printers to manufacture products directly from recycled plastic waste materials.

\section{Materials and Methods}

The main goal of this study is to compare the performance of parts manufactured between FFF and FGF on desktop 3D printers. The three main criteria that are evaluated are: (1) printability, (2) material properties, and (3) economic performance. The global experimental framework is presented in Figure 1.

\section{Materials}

Virgin and four different types of recycled PLA were used in this study:

1. Commercial PLA type 4043D from NatureWorks supplied in pellet form by NaturePlast (Caen, France) was used as the virgin plastic control for FGF.

2. Commercial recycled PLA filament was supplied by Formfutura $^{40}$ for recycled FFF.

3. Recycled PLA filament was manufactured in situ in fablab conditions from PLA wastes by using a desktop extruder $^{41}$ for recycled FFF.

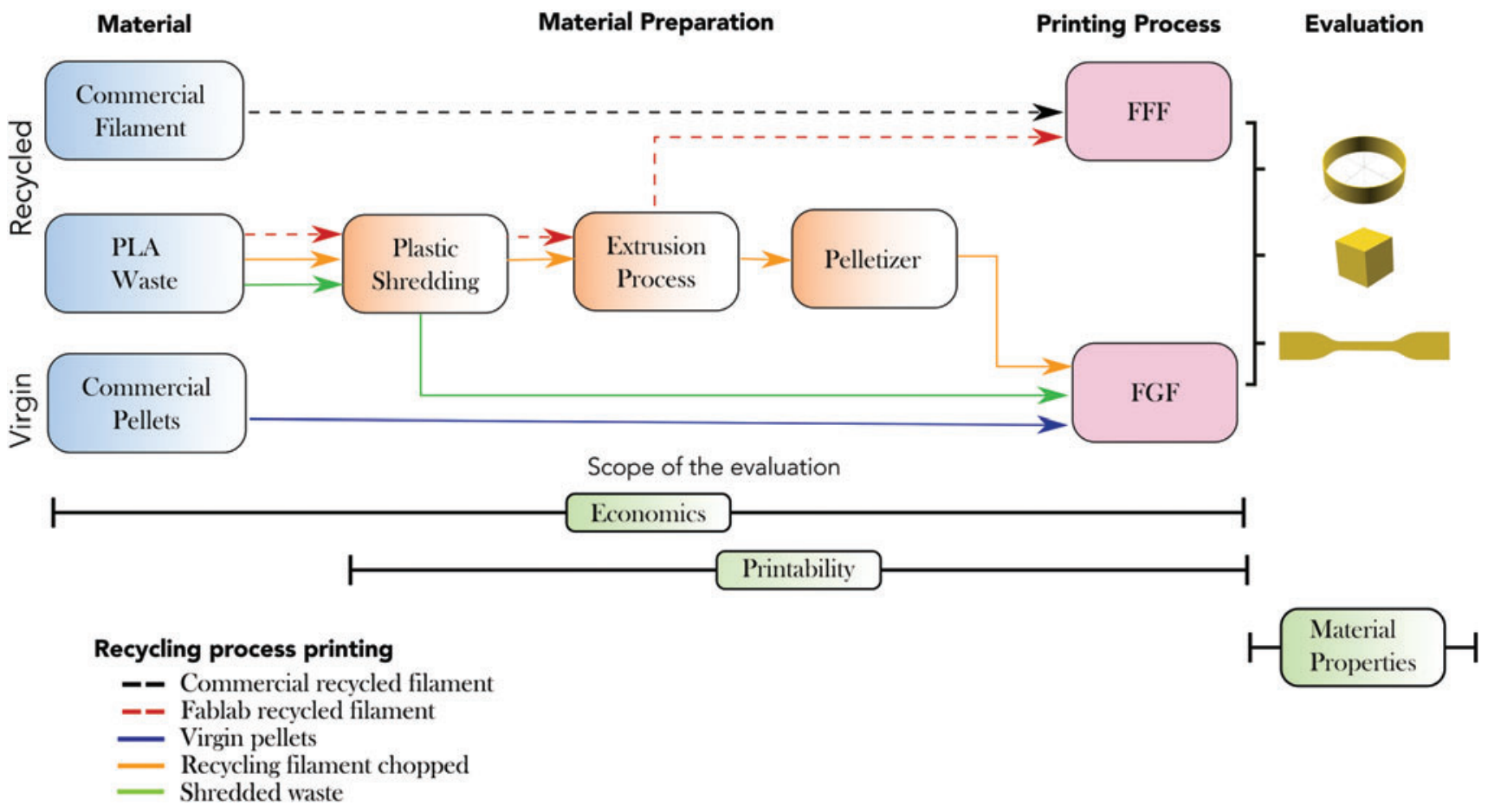

FIG. 1. Global framework of the experimentation. FFF, fused filament fabrication; FGF, fused granular fabrication; PLA, polylactic acid. Color images are available online. 
Table 1. Fixed Three-Dimensional Printing PARAMETERS FOR THE FUSED Filament FABRICATION and Fused Granular Fabrication Printers

\begin{tabular}{lccr}
\hline Parameter & $F F F$ & FGF & Units \\
\hline Layer height & 0.2 & 0.2 & $\mathrm{~mm}$ \\
Bed temperature & 60 & 60 & ${ }^{\circ} \mathrm{C}$ \\
No. of perimeters & 3 & 3 & \\
Top solid layers & 3 & 3 & \\
Bottom solid layers & 3 & 3 & \\
Fill density & $100 \%$ & $100 \%$ & \\
Fill & $0 / 90$ & $0 / 90$ & \\
Travel speed max & 140 & 40 & $\mathrm{~mm} / \mathrm{s}$ \\
Nozzle diameter & $0.5 / 0.8$ & 0.8 & $\mathrm{~mm}$ \\
\hline
\end{tabular}

FFF, fused filament fabrication; FGF, fused granular fabrication.

4. Pelletized feedstock was made from the recycled filament for FGF.

5. Finally, shredded PLA from 3D printing wastes was used directly for FGF.

\section{Material preparation}

In the material preparation phase, a professional cutting mill (Retsch SM 300) was used for the plastic shredding process. A single screw filament extruder (Noztek Xcalibur ${ }^{42}$ ) with a profile temperature of $160-190^{\circ} \mathrm{C}$ and an extruder nozzle diameter of $\phi 2 \mathrm{~mm}$ was used to manufacture the filament in a fablab conditions. The open source hardware (filawinder ${ }^{43}$ ) was used to control of the extruded filament, which was cooled by natural convection. Finally, a pelletizer was developed to cut the filament into small portions and was adapted for cutting portions adequate to the feeding size of the FGF printer. For the FFF process, the mean value of the diameter was used as a material quality indicator. For FGF, a size distribution analysis was performed to qualify the particle material. For this, an image analysis was performed to obtain the projected area from feedstock images by using the open-source ImageJ software. ${ }^{44}$

\section{Printing process: FFF and FGF}

For the FFF system, a derivative version of the RepRap machine Prusa I3 running Marlin firmware v1.1.9 was selected for the printing process. This machine is representative of the open-source RepRap-class 3D printers that dominate the open-source community. ${ }^{45,46}$ In the FFF process using
PLA recycled filaments, there has been characterization of the mechanical properties, including tensile, ${ }^{14}$ flexural, ${ }^{45}$ and rheological/thermal properties. ${ }^{47}$ These studies proved the technical feasibility of using recycled PLA for FFF. Based on this literature, Table 1 presents the print settings used for both PLA recycled filaments.

The FGF printer comprised a pellet extruder $\mathrm{kit}^{39}$ adapted to a commercial FFF printer (Créality CR-10S pro ${ }^{48}$ ) machine using a Marlin firmware v1.1.19. The pellet extrusion kit uses an auger screw with a diameter $\phi 8 \mathrm{~mm}$, cartridge heater $50 \mathrm{~W}-$ $24 \mathrm{~V}$, and nozzle diameter $\phi 0.8 \mathrm{~mm}$ that mixes and extrudes the melted material. The hot end of the FFF printer was adapted by replacing the pellet extruder prototype as shown in Figure 2. After the mechanical assembly was made, the first experimental tests were carried out to adapt the machine to the new parts and calibrate the formation of an extruded filament by using virgin PLA pellets. The extrusion factor was changed to calibrate the rotation of the screw extruder.

Printing parameters for FFF are well known and defined for PLA. For the FGF printing, however, a detailed means of determining print parameters was followed. Each of the material pellets was loaded into the hopper, and the initial set temperature was the same for FFF. However, unlike the FFF machine where only the nozzle is heated to fuse the filament, the experimental set-up needed higher temperatures to obtain a homogeneous melt and a steady flow. Thus, the temperature was gradually raised in increments of $5^{\circ} \mathrm{C}$ to have stable traces of extruded filament. Here, the virgin PLA pellets was taken as the best-case scenario given the fact that the channel depth of the auger screw was adequate for the pellet size, pushing down the materials already melted in the heated zone of the extruder. Also, the spherical form of virgin flakes enables a reduced contact area at the beginning of the feeding, which reduces the probability of feeding and clogging issues. Moreover, the consistent repeatability of the size distribution helps to have a reduced material variation in the printing process. Experimental changes in the firmware for the extruder motor (steps-per-unit -M92-) were tested to provide adequate material flow rate for the virgin pellets. The value was changed from 140 (initial configuration for Creality machine), in increments of 100 until 540. Regular printed values of extrusion for virgin pellets were found at 340 . The pelletized material has a well-defined geometrical shape (small cylinders) and provides an effect similar to the PLA granules in terms of reduced clogging. Likewise, the firmware value of the

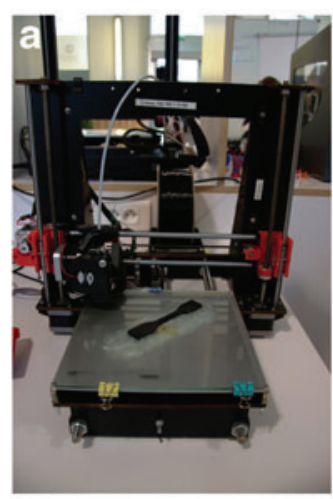

FFF printer
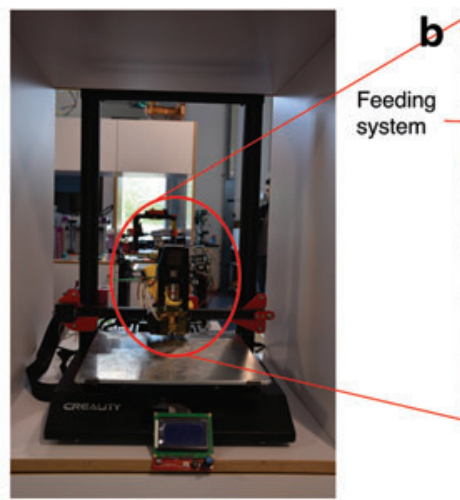

Extrusion motor \& gear box

Coupling

Cooliing unity

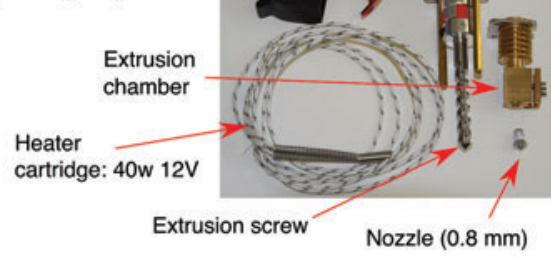

FGF printer

FIG. 2. (a) FFF and (b) FGF printers used in the experimentation. Color images are available online. 
extruder motor was adapted for pelletized material and an extrusion factor of 1000 was used given the reduction of the size distribution of the pelletized material. However, shredded PLA, because of the random shape and greater size variability, often caused an impasse, feeding and clogging issues.

The experimental trials showed an important influence of the temperature in the printing process for the shredded material. In the transition state, the conduction heat through the extruder screw section did not affect the feeding of material. However, in steady state of the printing process, the temperature gradient between the nozzle and the entry of feeding systems caused lumps of melted particles, which blocked the feeding and generated defects in the printed objects. Also, due to the variability of form of the shredded material, the feeding process by only gravitational forces resulted in variations of the extruded material during the printing process. Therefore, experimental adjustments were made from the original kit extruder based on community improvements ${ }^{49}$ to adapt a fan device at the beginning of the screw and insulate the heater chamber.

After appropriate cooling was enabled, an exploratory analysis using factors such as nozzle temperature, fan speed, and print speed were selected with different levels to print the recycled material by using the slicer to control the variables. The nozzle temperature was changed from $220^{\circ} \mathrm{C}$ to $230^{\circ} \mathrm{C}$. Fan speed was progressively increased from 40 to $100 \mathrm{~mm} / \mathrm{s}$. Then, the printing speed was altered from $30 \%$ to $100 \%$ to explore combinations of linear speed and extrusion speed. The purpose was to find usable settings to obtain a reproducible printing process with the three types of FGF materials. Objects were printed to validate the wall thickness and accuracy on the $\mathrm{X}, \mathrm{Y}$, and $\mathrm{Z}$ axes. Stable printings were found by using a temperature of 225 and a fan speed of $40 \mathrm{~mm} / \mathrm{s}$. Based on these conditions, printed objects were obtained for each material to validate the $\mathrm{X}, \mathrm{Y}$, and $\mathrm{Z}$ accuracy and the wall thickness. Percentage error was found by using three repetitions for each object and material for the FGF PLA feedstocks. For both the FFF and FGF printing, all slicing was done with PrusaSlic3r v.2.1.0+.

\section{Evaluation}

Printability. The creation of index of "printability" (or "additive manufacturing index") is one important research path to clarify the potential of a material to be used in the $3 \mathrm{D}$ printing technologies. ${ }^{50}$ This index associates consideration such as the processability/formability as feedstock for specific additive manufacturing (AM) technology, final properties (mechanical, geometrical), and postprocessing requirements, which finally represent an aid-decision tool for practitioners of AM technologies. First, size distribution and diameter measurements of the feedstock materials were used to evaluate the quality of each feedstock. Next, an experimental screening was made to determine adequate printing temperature, the extrusion factor, and the fan's speed in the direct extrusion machine. Basic prints of a single-walled vase test and cube $^{24}$ objects were made to validate the printing parameters for each type of material granulometry. Digital calipers $( \pm 0.01 \mathrm{~mm})$ were used to validate the printed wall thickness and reliability. Once the print was completed, the specimen's mass was recorded and compared with the theoretical mass of the component by using the specific density of the plastic to prove the reproducibility of the printing.
Material properties. Eight tensile test samples were printed for each type of material according to ASTM D638 Type IV to evaluate tensile strength, strain, and elastic modulus among the five material configurations. The weight and the cross-sectional dimensions of the sample were measured before the tensile test. An open-source slicer was used to obtain the G-code for each material by using a horizontal alignment configuration for the $\mathrm{X}-\mathrm{Y}$ plane. The samples were printed indoors with a controlled temperature with $100 \%$ infill and a $0 / 90$ pattern with respect to the long axis of the tensile bars. ${ }^{51}$ The tests were performed on the ZWick 1476 machine by using an extensometer of $20 \mathrm{~mm}$.

Economic performance. A comparison was made between the cost to print $1 \mathrm{~kg}$ of material $\left(U_{c}\right)$ by the FGF and the FFF printers. The economic analysis was made considering the material acquisition costs $\left(M_{c}\right)$ and the operating time and energy consumption $\left(E_{c}\right)$ of each machine. The data related to energy consumption for each printing route were collected through a smart power plug device ${ }^{52}( \pm 0.001 \mathrm{kWh})$ where the consumption data are stored. The average consumption during the printing process of the mechanical was used in the analysis. Equation 1 was used to compute the cost of each 3D-printed product component $U_{c}$ :

$$
U_{c}=M_{c}+E_{c} \quad[€ / k g]
$$

where the energy cost per $\mathrm{kg}\left(E_{c}\right)$ is calculated with Equation 2:

$$
E_{c}=E \times E_{p} \quad[€ / k g]
$$

Here, $E_{p}[€ / \mathrm{kWh}]$ represents the price of local energy and $E$ $[\mathrm{kWh} / \mathrm{kg}]$ represents the energy consumed. Equation 3 estimates the cost of preparation per $\mathrm{kg}$ of the shredded waste plastic $S_{c o}$ $[€ / \mathrm{kg}]$ in a fablab context. It was considered the shredder capacity $S_{c a}[\mathrm{~kg} / \mathrm{h}]$, the energy consumed by the machine in $1 \mathrm{~h}$ $S_{\text {cons }}[\mathrm{kWh} / \mathrm{h}]$, and the local energy cost $E_{p}[€ / \mathrm{kWh}]$.

$$
S_{c \mathrm{o}}=\frac{S_{\text {cons }} \times E_{p}}{S_{c a}} \quad[€ / k g]
$$

The cost of fabrication of recycled filament $F_{F}[€ / \mathrm{kg}]$ was considered as a function of the shredded waste cost $S_{c o}$ (equation 3) and energy cost of the filament extrusion machine $\operatorname{Ext}_{c}[€ / \mathrm{kg}]$, as illustrated in Equation 4:

$$
F_{F}=S_{c o}+E_{c} \quad[€ / k g]
$$

where the energy cost of the filament extruder machine per $\mathrm{kg}$ $E x t_{c}[€ / \mathrm{kg}]$ takes into consideration the capacity of the extruder machine $E x t_{\text {cap }}[\mathrm{kg} / \mathrm{h}]$, the energy consumption of the extruder $E x t_{\text {cons }}[\mathrm{kWh} / \mathrm{h}]$, and the local energy cost $E_{p}[€ /$ $\mathrm{kWh}$ ] as presented in Equation 5:

$$
\operatorname{Ext}_{c}=\frac{\operatorname{Ext}_{\text {cons }} \times E_{p}}{\operatorname{Ext}_{\text {cap }}} \quad[€ / \mathrm{kg}]
$$

\section{Results \\ Printability}

Figure 3 presents the quality of the feedstock material for both the FFF and FGF processes. The quality of the filament 
is observed to be an important factor in printing for FFF. ${ }^{53}$ Figure $3 \mathrm{a}$ presents the diameter variation among the commercial recycled and fablab in situ manufactured filament. Figure $3 \mathrm{~b}$ plots the digital image analysis of the size distributions for the three types of FGF materials: virgin pellets, pelletized recycled filament, and shredded PLA.

The commercial recycled filament presented a visibly more homogeneous composition (with fewer visible defects such as deformations and bubbles) and a more uniform diameter along the entire length $1.71 \mathrm{~mm} \pm 2.07 \%$, resulting in fewer printing errors (clogging, lack of material, excess material). The fablab filament resulted in a diameter of $1.53 \mathrm{~mm} \pm 6.5 \%$, which is a more significant variability. In addition, it has more visible flaws along the entire length, which translated into more printing problems.

For FGF, the virgin PLA presented the largest and most uniform size distribution of $13.5 \mathrm{~mm}^{2} \pm 6.9 \%$. The pelletized and shredded material were $36 \%$ and $74 \%$ smaller than virgin ones, respectively.

Once the clogging issues were reduced as described in the Materials and Methods section, Figure 4 presents the visual quality of the printed material by using different extruder motor steps-per-unit extrusion factors (from 700 to 1500) and the influence of the cooling system by using shredded materials, which was the most challenging.

The results of the exploratory analysis to find print parameters for the recycled material by using factors such as nozzle temperature, fan speed, and print speed is illustrated in Figure 5. For virgin material, the extrusion speed was the same as in FFF, giving an average deposition rate of about $0.33 \mathrm{gr} / \mathrm{min}$. However, for pelletized and shredded material to reduce the probability of feeding issues even more, the extrusion speed factor was reduced to obtain approximative deposition rate values of about 0.31 and $0.26 \mathrm{gr} / \mathrm{min}$, respectively.

Table 2 presents the percentage error by using three repetitions for each object and material for the experiments used to determine the printing parameters for the FGF PLA feedstocks.

\section{Mechanical testing results}

Figure 6 shows the comparison of mechanical properties for the FFF and FGF process. The mean average of the tensile strength for the FFF technique was $51.67 \pm 1.6 \mathrm{MPa}$ (nozzle $0.5 \mathrm{~mm}$ ) and $57.81 \pm 1.8 \mathrm{MPa}$ (nozzle $0.8 \mathrm{~mm}$ ) for commercial

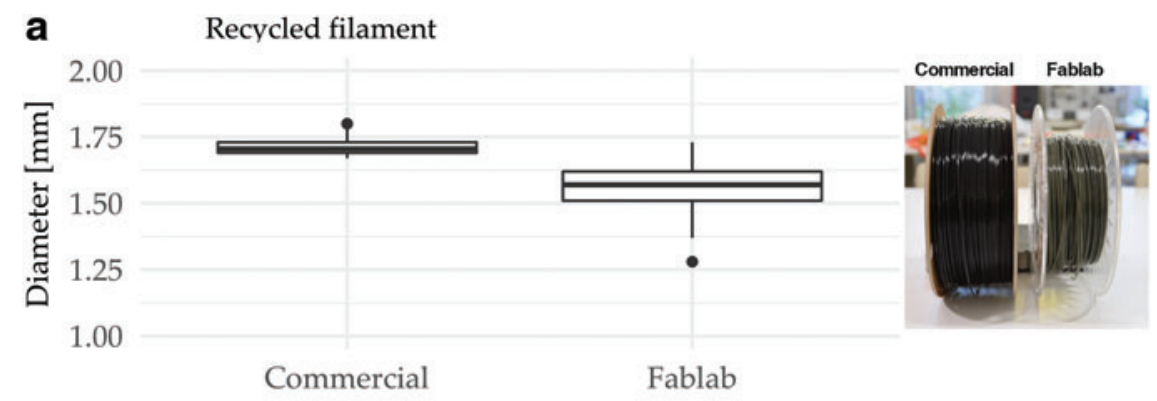

b

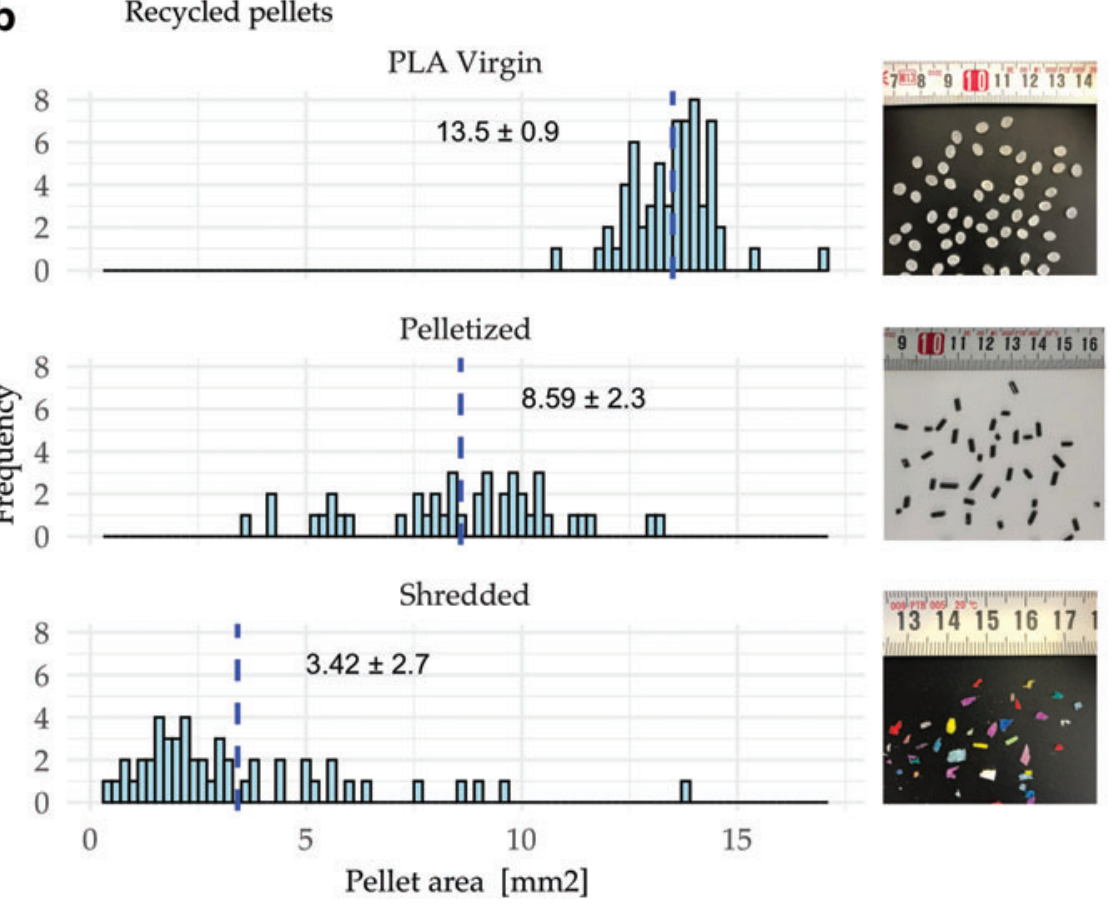

FIG. 3. Feedstock analysis for FFF and FGF. (a) presents the diameter difference for the FFF feedstock; (b) shows the size distribution in $\mathrm{mm}^{2}$ of the virgin pellets, pelletized recycled filament, and shredded PLA for the FGF process. Color images are available online. 


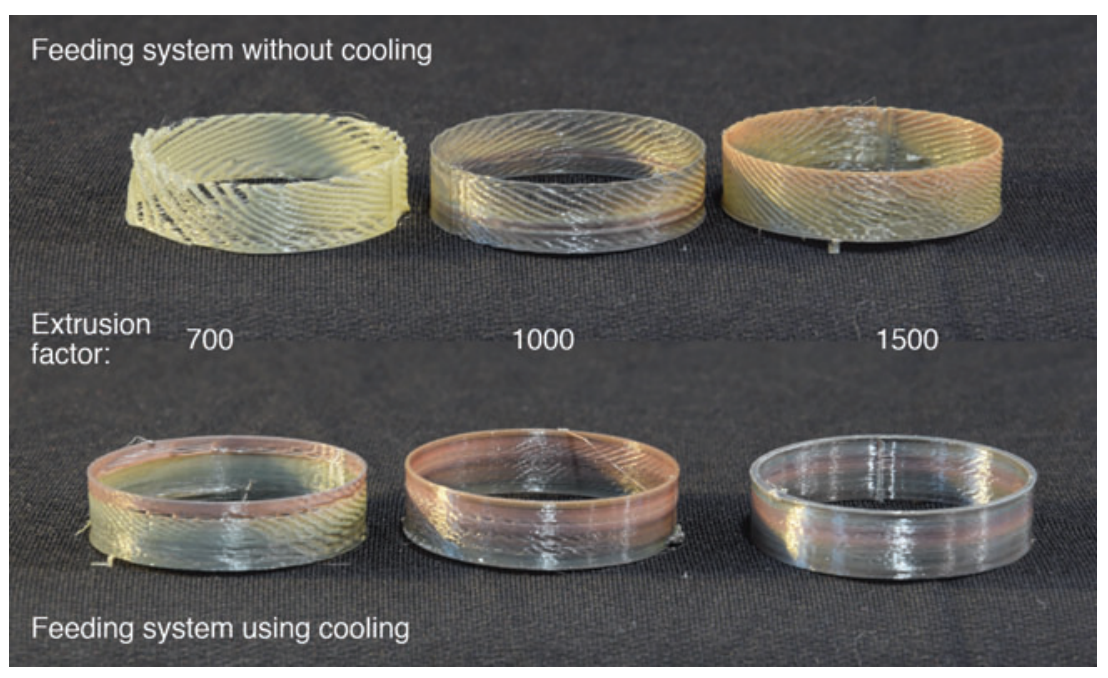

FIG. 4. Printability of shredded PLA materials. Color images are available online.

recycled filament. It was found to be $50.84 \pm 5.8 \mathrm{Mpa}$ (nozzle $0.5 \mathrm{~mm}$ ) and $58.3 \pm 5.3 \mathrm{Mpa}$ (nozzle $0.5 \mathrm{~mm}$ ) for fablab filament. This represents an increase of about $11 \%$ in mean. Kuznetsov et al. ${ }^{54}$ identified an increase of flexural strength due to the nozzle diameter, which could be seen in our results. The average values between both FFF processes using the same nozzle showed no large discrepancies and were consistent with the literature of recycled filament by using the chosen layer thickness and pattern orientation. ${ }^{53,55}$ However, the commercial filament presented less variation compared with the localmanufactured filaments, which can be influenced by the fact that the local filament presents a greater standard deviation of the diameter, besides visible extrusion defects along the entire length.

Regarding the FGF process, one main conclusion is that the tensile strength value for FGF using the recycled material has comparable performance to the traditional FFF technique. Indeed, one interesting result is that the values were about $10 \%$ on average higher than the FFF printer using the $0.5 \mathrm{~mm}$ nozzle. The average tensile strengths were $59.1 \pm 1.6 \mathrm{MPa}$ (virgin pellets), $60.3 \pm 4.5 \mathrm{MPa}$, and $52.7 \pm 6.4 \mathrm{MPa}$ material. The samples with the same nozzle value presented no statistical differences in the the tensile strength between FFF and FGF, with $95 \%$ confidence giving a $(-2.6,3.7) \mathrm{MPa}$ confidence interval. The virgin material presented the most homogeneous form and size, which could explain the reproducibility of the results in terms of tensile strength. It seems that the mean strain values were considerably lower (22\%) to FFF samples using the same nozzle. The pelletized material presented a comparable tensile strength, but a mean strain value that was $5.1 \%$ lower than the virgin samples. On the other hand, shredded materials resulted in the lowest mean tensile value. Nevertheless, it is worth noting that these results are encouraging because even with the difference in the size granulometry of the feedstock for FGF, the mechanical properties remain comparable to the FFF and the FGF (virgin and pelletized forms). It means that the direct printing process of directly shredded PLA wastes is technically feasible even
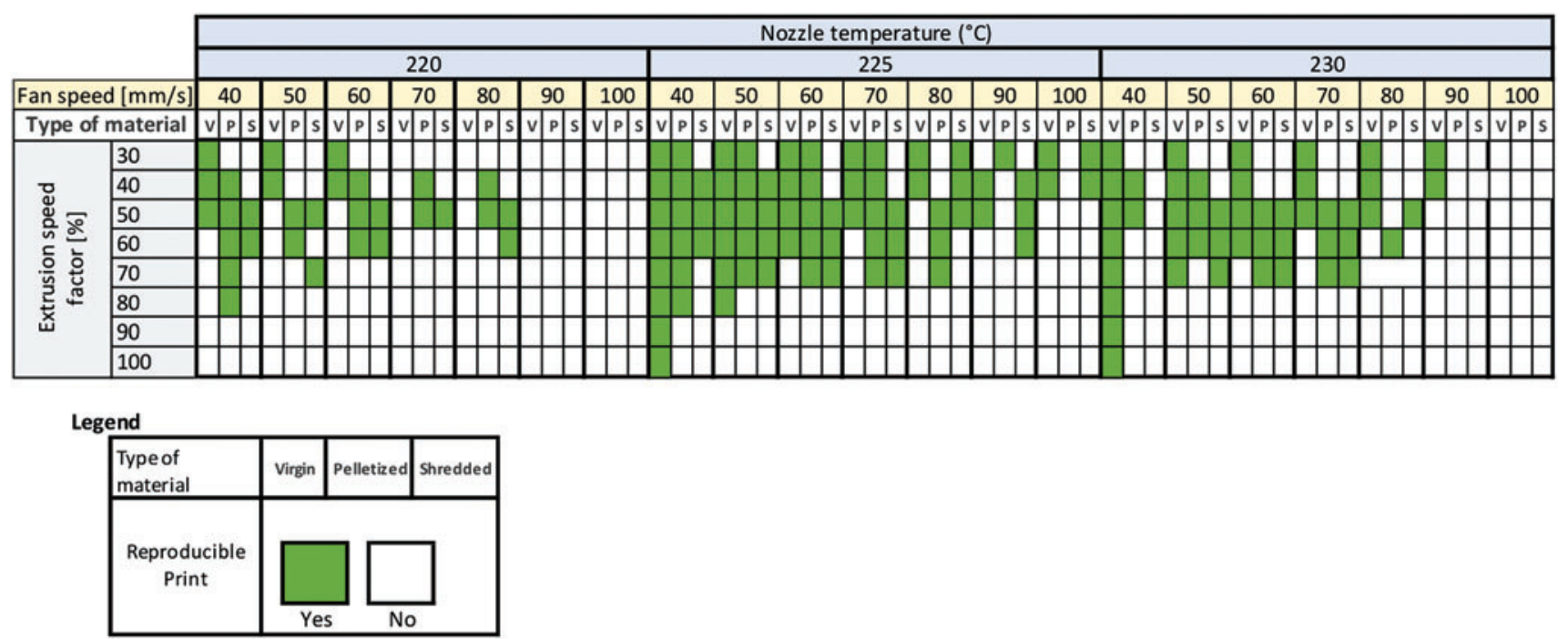

FIG. 5. Determining print settings for FGF. Color images are available online. 
Table 2. Percentage Error in the Printing Tests for Fused Granular Fabrication Materials

\begin{tabular}{|l|l|l|l|}
\hline Measures & Virgin & Pelletized & Shredded \\
\hline $\mathrm{X}$ & $0.25 \%$ & $2.4 \%$ & $3.7 \%$ \\
\hline $\mathrm{Y}$ & $0.32 \%$ & $1.85 \%$ & $3.45 \%$ \\
\hline $\mathrm{Z}$ & $0.12 \%$ & $0.7 \%$ & $1.5 \%$ \\
\hline Wall thickness & $12.5 \%$ & $2.5 \%$ & $5 \%$ \\
\hline
\end{tabular}

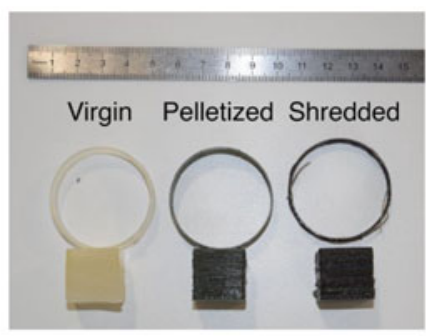

Color images are available online.

with the DIY kit direct extrusion system used here. This reduces the step process to recycle waste plastic with AM, reducing the degradation that the waste material forms in the fabrication of the filament.

However, it is clearly observed that the size particle variation causes a greater variation of material deposited during printing and the resultant mechanical properties of the printed object. A laboratory weighing balance was used to take the weight measurements of the tensile samples to evaluate whether the printed sample corresponds to the theoretical weight $(7.59 \mathrm{gr})$ from the CAD model as recommended by Tanikella et al. ${ }^{56}$ Figure 7 presents the specific tensile strength per sample type. It can be observed that the commercial recycled filament and commercial pellets are the most reproducible, and they influence the reproducibility of the mechanical properties. More research is needed to qualify the deposition of the direct extrusion systems.

\section{Economic results}

Acquisition cost. The cost of the FFF derivative version of the RepRap machine Prusa I3 ${ }^{57}$ was $€ 190.00$, with some modifications adding to a total of $€ 230.00$. For the FGF prototype, the Creality CR-10S pro ${ }^{48}$ was priced at $€ 539.10$ plus the pellet extruder kit $^{39}$ with a cost of $€ 289.00$, leading to a total cost of $€ 828$.10. It should be noted that the Prusa I 3 clone was a DIY considerably more challenging to build compared with the Creality printer requiring a time investment of $\sim 24 \mathrm{~h}$ for one person with technical competence to assemble. In addition, the working volumes $(220 \times 220 \times 180 \mathrm{~mm}$ and $300 \times 300250)$ and the materials of the framework (Laser-cur MDF board and aluminium) for Prusa and Creality, respectively, make the differences in the cost of the equipment. Thus, for the experimental systems used here, the capital investment for the FGF 3D printer was $3.6 \times$ the cost of the FFF; however, for comparable $3 \mathrm{D}$ printers, it would have been about $1.5 \times$.

Raw materials cost. Five types of materials were used to make the objects in mechanical tests and their costs result from the formulas presented in Economic performance:

1. The cost for the recycled commercial filament was $24.96 € / \mathrm{kg}^{58}$

2. For the acquisition of virgin PLA pellets the cost was $8.50 € / \mathrm{kg}^{59}$

3. For the shredded waste the cost was calculated considering the free purchase of raw material (garbage already sorted and cleaned from Fablab), and free labor at Fablab. The use of energy by the machine to grind the material $\left(M_{c h}\right)$ was validated at $0.1383 \mathrm{kWh} / \mathrm{h}$ and the shredding capacity $\left(S_{c a}\right)$ of $4.35 \mathrm{~kg} / \mathrm{h}$ in a similar job done with acrylonitrile butadiene styrene ${ }^{18}$ and at a local energy cost of $0.153 € / \mathrm{kWh} .{ }^{60}$ The final cost was $0.004855 € / \mathrm{kg}$.

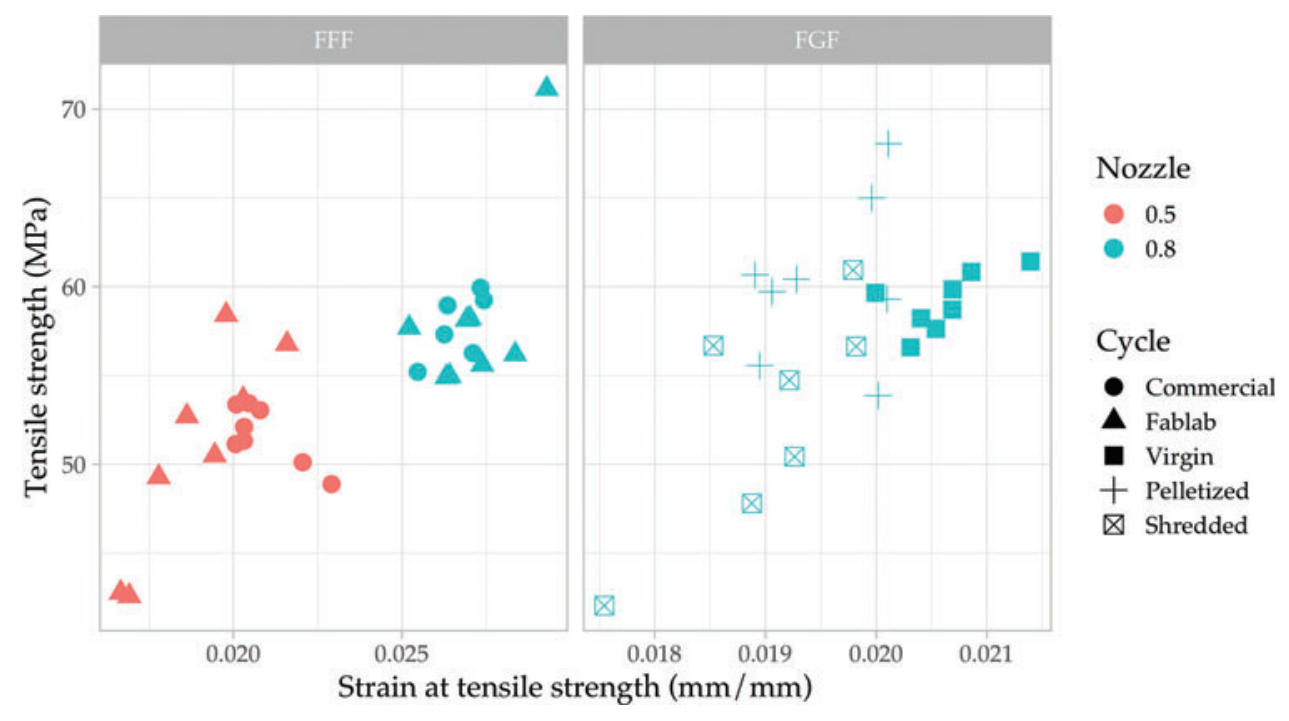

FIG. 6. Tensile strength versus strain at tensile strength of the recycled printed samples using FFF and FGF. Color images are available online. 


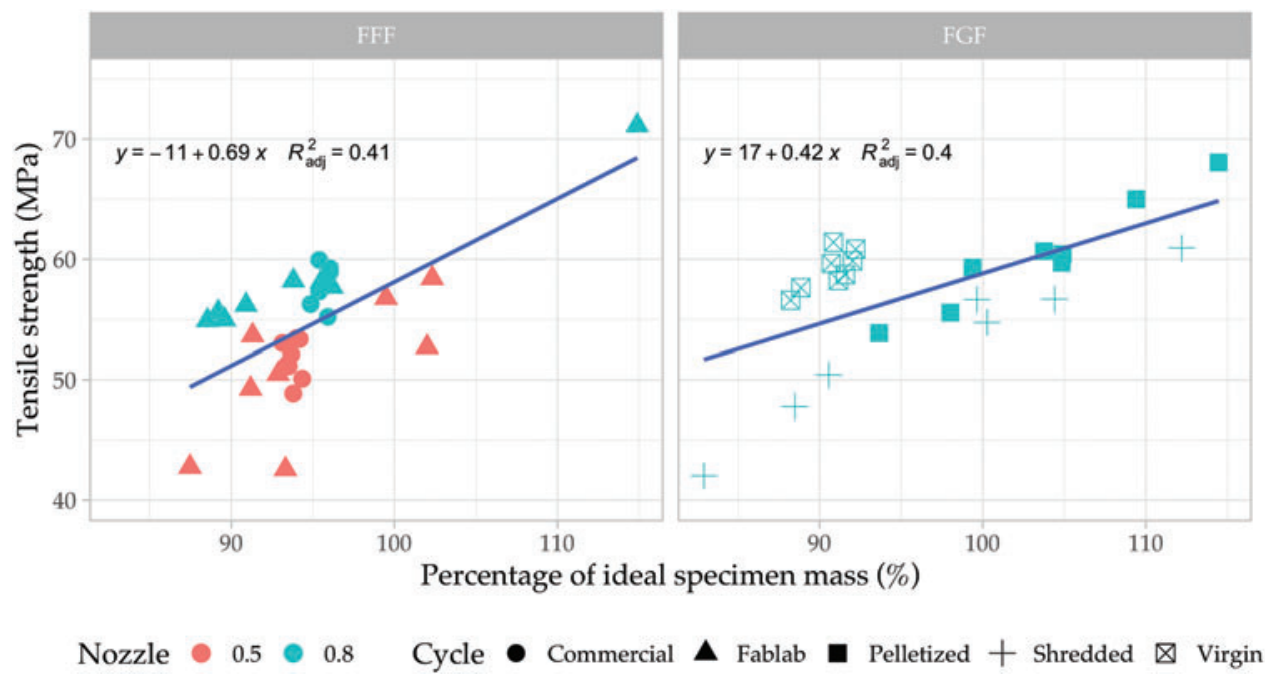

FIG. 7. Tensile strength as a function of sample mass percentage for FFF and FGF mechanical samples. Color images are available online.

4. The cost of the filament at fablab conditions considered the same cost of shredded material adding the energy cost of the filament manufacturing. Experimental tests estimated the extrusion capacity Ext cap $_{\text {of }}$ of $1.075 \mathrm{~kg} / \mathrm{h}$, consuming $0.25 \mathrm{kWh}$. This leads to a cost for filament cost of $0.0404 € / \mathrm{kg}$.

5. This value of $0.0404 € / \mathrm{kg}$ was also taken for the pelletized filament under fablab conditions.

Three-dimensional printers energy cost. The cost of 3D printing is directly linked to the time and energy consumed by the printer. For the FFF printer, the energy use was calculated at $0.096 \mathrm{kWh}$ per hour of printing. For the FGF, to maintain print quality, the time has varied according to the material, consuming an average of $0.12 \mathrm{kWh}$ per hour of printing.

Table 3 was constructed to summarize the cost $/ \mathrm{kg}$ and display a projection of the quantities produced to clarify the economics of the different material choices.

Table 3 shows that for the 3D printers used in this experiment making recycled filament as a user immediately reduces the costs of printing per $\mathrm{kg}$ by about $26 €$, which is nearly the cost of the recycled commercial filament. Note: the capital cost of the recyclebot was not taken into account. The difference in the capital costs of the FGF compared with the FFF indicated that for printing pellets $34 \mathrm{~kg}$ of printing would need to occur to offset the machine costs as used here. This was reduced to $23 \mathrm{~kg}$ for the direct extrusion materials. If a similar machine was used, these print paybacks were reduced further to 16 and $11 \mathrm{~kg}$, respectively.

\section{Discussion}

The main goal of this work is to compare the technical feasibility of printing recycled material by using FFF and FGF techniques. The results of recycled FFF technique were considered as a base reference. There have been several studies using FGF to print recycled materials with large FGF machines. ${ }^{62}$ Here, a small printer was tested that could be an additional option to the well-established FFF general consumer market.

Considering the printability aspect, recent research starts exploring the modeling of the printing process in FGF. ${ }^{63}$ However, the development of methodologies to establish optimal parameter conditions for FGF technology correlating material type, size distribution, and printing conditions is a major path in the development and spread of this manufacturing technique. In the FFF technique, the liquifier dynamics ${ }^{64}$ enables researchers to understand the major parameters related to the deposition of the extruded lines. For FGF, there is not a

Table 3. Analysis Cost for $1 \mathrm{Kg}$ of Recycled Material

\begin{tabular}{|c|c|c|c|c|c|}
\hline Cost type & FFF commercial & FFF fablab & $D E$-virgin & DE_pelletized & DE-shredded \\
\hline Material $/ \mathrm{kg}$ & $25.96 €$ & $0.0404 €$ & $8.5 €$ & $0.0404 €$ & $0.004855 €$ \\
\hline Energy cost/h & $0.01469 €$ & $0.01469 €$ & $0.01836 €$ & $0.01836 €$ & $0.01836 €$ \\
\hline Printing $1 \mathrm{~kg}$ & $50 \mathrm{~h}$ & $50 \mathrm{~h}$ & $33 \mathrm{~h}$ & $43 \mathrm{~h}$ & $50 \mathrm{~h}$ \\
\hline Printing energy cost/kg & $0.7345 €$ & $0.7345 €$ & $0.6059 €$ & $0.7895 €$ & $0.918 €$ \\
\hline Total printing cost $/ \mathrm{kg}$ & $26.7 €$ & $0.7749 €$ & $9.106 €$ & $0.8298 €$ & $0.9226 €$ \\
\hline $\begin{array}{l}\text { No. of kg needed to offset capital cost } \\
\text { of exp. difference to commercial filament }\end{array}$ & NA & $0^{\mathrm{a}}$ & 34 & 23 & 23 \\
\hline $\begin{array}{l}\text { No. of kg needed to offset capital cost } \\
\text { of equiv. difference to commercial filament }\end{array}$ & NA & $0^{\mathrm{a}}$ & 16 & 11 & 11 \\
\hline
\end{tabular}

${ }^{a}$ Does not include the capital costs of the recyclebot machine that vary widely from commercial systems to DIY and even threedimensional printable systems. ${ }^{61}$

NA, not applicable. 
clear methodology to establish and this is an important aspect to understand the influence of the parameters in the printing process.

Considering the material properties, one important result is that the FGF approach would not compromise the mechanical resistance of the recycled printed parts. On the contrary, the experimental results shown here provided small improved properties at a lower cost. Regarding the economic aspect, using the FGF printer with virgin PLA pellets, there is a $65 \%$ reduction in printing cost per kilogram and a shorter production time compared with recycled commercial filaments, which is a non-negligible option. The results show that the main cost in 3D FFF printing is in the acquisition of filaments. However, the acquisition of recycled material filaments reduces the cost in relation to the acquisition of virgin material filaments, providing a reduction in the use of virgin raw material in 3D printing. The capital cost differences show that the reduced material costs from FGF are most attractive to heavy AM users (e.g., small- and medium-sized enterprises, fablabs, makerspaces, schools, libraries, etc.). It also appears clear from the results in Table 3 that the differences in the costs of and FFF and FGF will need to be reduced to accelerate the adoption of FGF for the smaller volume/year printing of the prosumer market.

These results suggest that an exploratory path regarding the plastic recycling with FGF is technically possible. There is a reduction in terms of process steps to treat waste material. However, the FGF process is not a mature technology. The development of this extrusion systems requires further study in terms of influence of the granulometry (size and form) in the printing process. These systems are designed for spherical and uniform shapes, which made the use of shredded waste material difficult. Opportunities arise in the possibility of using other types of recycled waste, including flexible and composite (plastic/plastic) materials as has been done on larger systems. Also, main factors such as polymer viscosity, which need to be controlled in the FGF process, are needed.

\section{Conclusions}

The results showed that the small FGF-printed components presented comparable mechanical performance, a time gain and significant economic savings over the FFF printing process. The use of virgin material on the FGF printer is an economic advantage regarding the traditional FFF technique, but to make up for the additional capital costs several $\mathrm{kg}$ of material must be printed. Moreover, the mechanical performance of directly shredded and printed material was shown to be comparable to FFF. These results are an important step to facilitate the distributing recycling approach, where prosumers themselves use their own recycled waste to fabricate their own products. The evaluation of geometric performance to analyze the accuracy of this technology is a relevant future research work.

\section{Funding Information}

This work was supported partly by the French PIA project "Lorraine Université d'Excellence" reference ANR-15-IDEX -04-LUE.

\section{Author Disclosure Statement}

No competing financial interests exist.

\section{References}

1. Bicket M, Guilcher S, Hestin M, et al. Scoping study to identify potential circular economy actions, priority sectors, material flows and value chains. Publications Office of the European Union. 2014. Available from https://www.eesc .europa.eu/resources/docs/scoping-study.pdf (last accessed October 13, 2019).

2. Raynaud J. Valuing plastics: The business case for measuring, managing and disclosing plastic use in the consumer goods industry. UNEP. 2014. Available from http://hdl.handle.net/ 20.500.11822/9238 (last accessed November 1, 2019).

3. Geyer R, Jambeck JR, Law KL. Production, use, and fate of all plastics ever made. Sci Adv 2017;3:e1700782.

4. Craighill AL, Powell JC. Lifecycle assessment and economic evaluation of recycling: A case study. Resour Conserv Recycl 1996;17:75-96.

5. Themelis N, Castaldi M, Bhatti J, et al. Energy and economic value of non recycled plastics (NRP) and municipal solid wastes (MSW) that are currently landfilled in the fifty states. Earth Engineering Center, Columbia University, New York. 2011.

6. Wittbrodt BT, Glover A, Laureto J, et al. Life-cycle economic analysis of distributed manufacturing with opensource 3-D printers. Mechatronics 2013;23:713-726.

7. Gwamuri J, Wittbrodt BT, Anzalone NC, et al. Reversing the trend of large scale and centralization in manufacturing: The case of distributed manufacturing of customizable 3-Dprintable self-adjustable glasses. Chall Sustain 2014;2:30-40.

8. Kietzmann J, Pitt L, Berthon P. Disruptions, decisions, and destinations: Enter the age of 3-D printing and additive manufacturing. Bus Horiz 2015;58:209-215.

9. Laplume A, Anzalone GC, Pearce JM. Open-source, selfreplicating 3-D printer factory for small-business manufacturing. Int J Adv Manuf Technol 2016;85:633-642.

10. Mai J, Zhang L, Tao F, et al. Customized production based on distributed $3 \mathrm{D}$ printing services in cloud manufacturing. Int J Adv Manuf Technol 2016;84:71-83.

11. Kreiger M, Pearce JM. Environmental life cycle analysis of distributed three-dimensional printing and conventional manufacturing of polymer products. ACS Sustain Chem Eng 2013;1:1511-1519.

12. Kreiger M, Pearce JM. Environmental impacts of distributed manufacturing from 3-D printing of polymer components and products. MRS Proc 2013;1492:85-90.

13. Baechler C, DeVuono M, Pearce JM. Distributed recycling of waste polymer into reprap feedstock. Rapid Prototyp J 2013;19:118-125.

14. Cruz Sanchez FA, Boudaoud H, Hoppe S, et al. Polymer recycling in an open-source additive manufacturing context: Mechanical issues. Addit Manuf 2017;17:87-105.

15. Kreiger MA, Mulder M, Glover AG, et al. Life cycle analysis of distributed recycling of post-consumer high density polyethylene for 3-D printing filament. J Clean Prod 2014;70:90-96.

16. Zhong S, Rakhe P, Pearce J. Energy payback time of a solar photovoltaic powered waste plastic recyclebot system. Recycling 2017;2:10.

17. Zhao XG, Hwang K-J, Lee D, et al. Enhanced mechanical properties of self-polymerized polydopamine-coated recycled PLA filament used in 3D printing. Appl Surf Sci 2018;441:381-387.

18. Zhong S, Pearce JM. Tightening the loop on the circular economy: Coupled distributed recycling and manufacturing with recyclebot and reprap 3-D printing. Resour Conserv Recycl 2018;128:48-58. 
19. Sells E, Bailard S, Smith Z, et al. RepRap: The replicating rapid prototyper: Maximizing customizability by breeding the means of production. In: Piller FT, Tseng MM (eds.) Handbook of Research in Mass Customization and Personalization (In 2 volumes). Singapore, Singapore: World Scientific, 2010; pp. 568-580.

20. Jones R, Haufe P, Sells E, et al. RepRap-The replicating rapid prototyper. Robotica 2011;29:177-191.

21. Bowyer A. 3D printing and humanity's first imperfect replicator. 3D Print Addit Manuf 2014;1:4-5.

22. Herzberger J, Sirrine JM, Williams CB, et al. Polymer design for 3D printing elastomers: Recent advances in structure, properties, and printing. Prog Polym Sci 2019;97:101144.

23. Cruz Sanchez FA, Lanza S, Boudaoud H, et al. Polymer recycling and additive manufacturing in an open source context: Optimization of processes and methods. In: Annual International Solid Freeform Fabrication Symposium, ISSF 2015. Austin, TX: University of Texas at Austin 2015; pp. 1591-1600.

24. Woern A, Byard D, Oakley R, et al. Fused particle fabrication 3-D printing: Recycled materials' optimization and mechanical properties. Materials 2018;11:1413.

25. Anderson I. Mechanical properties of specimens 3D printed with virgin and recycled polylactic acid. 3D Print Addit Manuf 2017;4:110-115.

26. Pakkanen J, Manfredi D, Minetola P, et al. About the use of recycled or biodegradable filaments for sustainability of $3 \mathrm{D}$ printing. In: International Conference on Sustainable Design and Manufacturing. Cham, Switzerland: Springer, 2017; pp. 776-785.

27. Tanney D, Meisel NA, Moore J. Investigating material degradation through the recycling of PLA in additively manufactured parts. In Proceedings of the 28th Annual International Solid Freeform Fabrication Symposium, Austin, TX, 2017; pp. 519-531.

28. Tian X, Liu T, Wang Q, et al. Recycling and remanufacturing of 3D printed continuous carbon fiber reinforced PLA composites. J Clean Prod 2017;142:1609-1618.

29. Volpato N, Kretschek D, Foggiatto J, et al. Experimental analysis of an extrusion system for additive manufacturing based on polymer pellets. Int J Adv Manuf Technol 2015;81:1519-1531.

30. Whyman S, Arif KM, Potgieter J. Design and development of an extrusion system for 3D printing biopolymer pellets. Int J Adv Manuf Technol 2018;96:3417-3428.

31. Liu X, Chi B, Jiao Z, et al. A large-scale double-stagescrew 3D printer for fused deposition of plastic pellets. $\mathrm{J}$ Appl Polym Sci 2017;134:45147.

32. Kumar N, Jain PK, Tandon P, et al. Extrusion-based additive manufacturing process for producing flexible parts. J Brazilian Soc Mech Sci Eng 2018;40:143.

33. Wang Z, Liu R, Sparks T, et al. Large-scale deposition system by an industrial robot (I): Design of fused pellet modeling system and extrusion process analysis. 3D Print Addit Manuf 2016;3:39-47.

34. Byard DJ, Woern AL, Oakley RB, et al. Green fab lab applications of large-area waste polymer-based additive manufacturing. Addit Manuf 2019;27:515-525.

35. Reich MJ, Woern AL, Tanikella NG, et al. Mechanical properties and applications of recycled polycarbonate particle material extrusion-based additive manufacturing. Materials (Basel) 2019;12:1642.

36. Horne R. Reprap development and further adventures in DIY 3D printing: No more filament?-Quest for a Uni- versal Pellet Extruder for 3D Printing. 2014. Available from: https://richrap.blogspot.com/2014/12/no-more-filament -quest-for-universal.html (last accessed July 3, 2019).

37. upe3D. Universal Pellet Extruder. Available from: http:// upe3d.blogspot.com/ (last accessed July 3, 2019).

38. Canessa E, Baruzzo M, Fonda C. Study of moineau-based pumps for the volumetric extrusion of pellets. Addit Manuf 2017; 17:143-150.

39. Mahormuniz. Pellet Extruder v3-MAHOR $\cdot X Y Z$. Available from: https://mahorxyz.wordpress.com/2016/05/02/pelletextruder/ (last accessed July 3, 2019).

40. FormFutura. ReForm. Available from: https://www.formfutura .com/shop/product/reform-rpla-off-black-975 (last accessed May 29, 2019).

41. Cruz Sanchez FA. Methodological proposition to evaluate polymer recycling in open-source additive manufacturing contexts (doctoral dissertation). Nancy and Metz, France: Université de Lorraine; 2016.

42. Noztek. Noztek Xcalibur. Available from: https://www .noztek.com/product/noztek-Xcalibur/ (last accessed July 10, 2019).

43. Filastruder. Filawinder. Available from: https://www.filastruder .com/products/filawinder (last accessed August 2, 2019).

44. Image J. Image processing and analysis in Java. Available from: https://imagej.nih.gov/ij/ (last accessed May 3, 2019).

45. Lanzotti A, Martorelli M, Maietta S, et al. A comparison between mechanical properties of specimens 3D printed with virgin and recycled PLA. Procedia CIRP 2019;79: 143-146.

46. Mercuri R, Meredith K. An educational venture into 3D Printing. In: 2014 IEEE integrated stem education conference. Austin, TX: University of Texas at Austin 2014; pp. 1-6.

47. Zhao $\mathrm{P}$, Rao $\mathrm{C}, \mathrm{Gu} \mathrm{F}$, et al. Close-looped recycling of polylactic acid used in 3D printing: An experimental investigation and life cycle assessment. J Clean Prod 2018;197: 1046-1055.

48. Creality. Creality CR 10s pro. Available from: https:// www.creality3dofficial.com/products/official-creality-cr-10spro-3d-printer (last accessed July 3, 2019).

49. Farnworth E. Universal pellet extruder V3 ZQ version. Available from: https://www.thingiverse.com/thing:3032188 (last accessed August 2, 2019).

50. Bourell D, Kruth JP, Leu M, et al. Materials for additive manufacturing. CIRP Ann 2017;66:659-681.

51. Laureto JJ, Pearce JM. Anisotropic mechanical property variance between ASTM D638-14 type i and type iv fused filament fabricated specimens. Polym Test 2018;68:294301.

52. MCL. Smart power plug. Available from: http://www.mclsamar .com/mcl_images/supports/dom-ps512/support_dom-ps512 .html (last accessed July 11, 2019).

53. Tymrak B, Kreiger M, Pearce J. Mechanical properties of components fabricated with open-source 3-D printers under realistic environmental conditions. Mater Des 2014;58:242246.

54. Kuznetsov VE, Solonin AN, Urzhumtsev OD, Schilling R, Tavitov AG. Strength of PLA components fabricated with fused deposition technology using a desktop 3D printer as a function of geometrical parameters of the process. Polymers 2018;10:313.

55. Wittbrodt B, Pearce JM. The effects of PLA color on material properties of 3-D printed components. Addit Manuf 2015;8:110-116. 
56. Tanikella NG, Wittbrodt B, Pearce JM. Tensile strength of commercial polymer materials for fused filament fabrication 3D printing. Addit Manuf 2017;15:40-47.

57. FNAC. Reprap prusa i3 DIY 3D. Available from: https:// www.fnac.com/mp38519066/Reprap-Prusa-i3-DIY-3DImprimante-Kits-110-240V-avec-8-GB-SD-Carte-NozzleAcrylique-Encadrement-2004LCD-Ecran-220-220-240mm -Zone-ABS-PLA-HIP-PP-Wood-Filament/w-4?oref $=42500$ e08-72ea-a48c-0647-4698a28208f3\%7B/\&\%7DOrigin= CMP\%7B/_\%7DGOOGLE\%7B/_\%7DM (last accessed August 2, 2019).

58. Filimprimante3D. Filament recyclé-Filimprimante3D. Available from: https://www.filimprimante3d.fr/87-filament -recycle (last accessed July 15, 2019).

59. Reprapworld. Natureworks Ingeo 4043D PLA pellets (1 kg) | 3D printing experts | reprapworld.fr. Available from: https://reprapworld.fr/products/filament/pellets/natureworks _ingeo_4043d_pla_pellets_1_kg/ (last accessed July 15, 2019).

60. Fournisseurseletricite. French energy supplier: Price per kWh of electricity in 2019 (in French). Available from: https://www.fournisseurs-electricite.com/guides/prix/kwhelectricite (last accessed July 16, 2019).
61. Woern AL, McCaslin JR, Pringle AM, et al. RepRapable recyclebot: Open source 3-D printable extruder for converting plastic to 3 -D printing filament. HardwareX 2018;4:e00026.

62. Shah J, Snider B, Clarke T, et al. Large-scale 3D printers for additive manufacturing: Design considerations and challenges. Int J Adv Manuf Technol 2019;104:3679-3693.

63. Khondoker MAH, Sameoto D. Direct coupling of fixed screw extruders using flexible heated hoses for FDM printing of extremely soft thermoplastic elastomers. Prog Addit Manuf 2019;4:197-209.

64. Bellini A, Guceri S, Bertoldi M. Liquefier dynamics in fused deposition. J Manuf Sci Eng 2004;126:237.

Address correspondence to:

Fabio A. Cruz Sanchez

ERPI

Université de Lorraine

8 rue Bastien Lepage, BP647

F-54000 Nancy

France

E-mail: cruzsanc1@univ-lorraine.fr 